\title{
Giant magnetization of a superconductor-two-dimensional electron gas-superconductor structure
}

\author{
I.A. Romanovsky ${ }^{1}$, E.N. Bogachek ${ }^{1}$, I.V. Krive ${ }^{2,3}$, and Uzi Landman ${ }^{1}$ \\ ${ }^{1}$ School of Physics, Georgia Institute of Technology, Atlanta, Georgia 30332-0430 \\ E-mail: igor.romanovsky@gmail.com \\ ${ }^{2}$ B. Verkin Institute for Low Temperature Physics and Engineering of the National Academy of Sciences of Ukraine, \\ 47 Lenin Ave., Kharkov 61103, Ukraine \\ ${ }^{3}$ Department of Physics, Göteborg University, SE 41296 Göteborg , Sweden
}

Received April 23, 2008

\begin{abstract}
Superconductivity induced phase-controlled mesoscopic magnetic effects in a two-dimensional electron gas that bridges two superconducting reservoirs are investigated. Giant paramagnetic response of the junction, occuring at certain values of the phase difference of the order parameter, is predicted. A geometrically similar system, consisting of a graphene ribbon stretched between two superconducting leads, is also considered. The magnetic effects in this system are found to be small and the difference between the magnetic properties of the two systems is discussed.
\end{abstract}

PACS: 73.63.Nm Quantum wires;

74.78.Na Mesoscopic and nanoscale systems;

74.45.+c Proximity effects; Andreev effect; SN and SNS junctions;

74.25.Bt Thermodynamic properties;

74.25.Ha Magnetic properties.

Keywords: supercurrent, magnetization, Andreev levels, graphene.

\section{Introduction}

Low-dimensional mesoscopic systems exhibit a number of properties which are of fundamental scientific interest, as well as some that may offer opportunities for the use of such systems in future miniaturized electronic devices. The new behavior exhibited by systems in this size regime made them the subject of extensive theoretical and experimental research over the past two decades.

Quantum wires (QW), are electric conductors with lateral (transverse) dimensions reduced such that the motion of the charge carriers in the wire becomes quantized. Quantum wires can be fabricated in many different ways. One of the common methods for the creation of QWs is the gate voltage method applied to semiconducting heterostructures with a two-dimensional electron gas $(2 \mathrm{DEG})$ at the interface $[1,2]$. Another often used method is the controlled break junction technique (CBJ). In this case a contact is pulled apart (for example, separating a contact formed between a tip and a surface) [3], or bent [4], in such a way that at the point where it breaks one gets a very narrow junction with a diameter comparable to the wavelength of the electrons at the Fermi level, resulting in conductance quantization $[1,2,5,6]$ and/or force oscillations $[3,4]$ that emerge upon continuous pulling of the contact. Quantum wires can also be made from quasitwo-dimensional conductors, such as graphene, by restricting the planar motion of the electrons in one of the two planar directions through the formation of sufficiently narrow ribbons [7].

Magnetic field effects in three-dimensional quantum wires connecting two superconductors have been studied previously in [8], where the phenomenon of giant magnetization oscillations was predicted. Here we focus on mesoscopic magnetic effects of superconducting-normal-superconducting (SNS) constrictions, shown schematically in Fig. 1, where the narrow (normal) part that connects the two bulk superconductors is a quasi-two-dimensional conductor. We consider two cases: (i) a superconductortwo-dimensional electron gas-superconductor (S/2DEG/S) junction and (ii) a superconductor-graphene monolayer-superconductor $(\mathrm{S} / \mathrm{GM} / \mathrm{S})$ junction. Both types of 


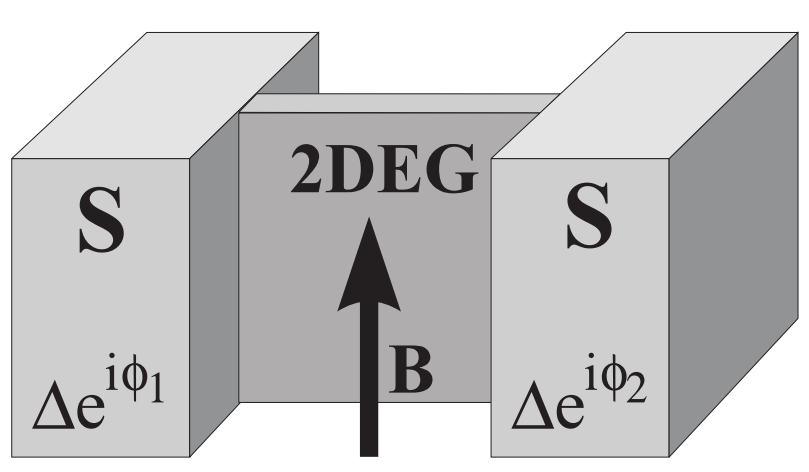

Fig. 1. A schematic description of the superconductor-2DEG-superconductor junction. Two superconductors with different phases of the order parameter are connected via a two-dimensional normal electron gas, or, alternatively, a narrow ribbon of graphene. A magnetic field is applied locally between the superconductors, parallel to the plane of the $2 \mathrm{D}$ conductor and is taken to be negligibly weak near the SN interfaces. A similar system has been considered in Ref. 14, where the effects of Zeeman splitting and spin-orbit interaction on the Josephson current were studied.

systems can be realized experimentally (see, e.g., [9-12]).

In the following section we consider the $\mathrm{S} / 2 \mathrm{DEG} / \mathrm{S}$ system. We investigate the influence of the barriers and the carrier effective mass differences at the interfaces between the semiconductor and superconductor on the Andreev levels and calculate the magnetization. In Sec. 3 we discuss the differences between the S/2DEG/S junction and $\mathrm{S} / \mathrm{GM} / \mathrm{S}$ junction, and explain the smallness of the magnetic field effects in the $\mathrm{S} / \mathrm{GM} / \mathrm{S}$ junction. We summarize our results in Sec. 4 .

\section{Magnetic effects in S/2DEG/S junctions}

In this section we consider a $\mathrm{S} / \mathrm{N} / \mathrm{S}$ contact made from a semiconductor heterostructures, that bridges two bulk superconductors. The difference in the electron band structure of the InAs, $\operatorname{In}_{x} \mathrm{Ga}_{1-x}$ As and $\mathrm{In}_{x} \mathrm{Al}_{1-x}$ As semiconductors leads to the formation of a narrow $\left(\sim \lambda_{F}\right)$ potential well in the InAs layer [13]. As a result, some of the conduction electrons of the semiconductors are trapped in this well, forming a two-dimensional electron gas. The quantum wires are fabricated by restricting the lateral dimensions of the two-dimensional electron gas in the semiconductor heterostructure through the use of electrostatic voltage gates. The potential of the electric field of the gates forms a bottleneck whose size in the narrowest part can be reduced to reach the order of several Fermi wavelengths. In- side this narrow region motion of the electrons in the directions that are transverse to the constriction axis is highly quantized and, consequently, at low temperatures only few transverse modes are populated and can conduct a current.

Since the motion of the electrons in the transverse direction is quantized, the motion of the electrons in each transverse mode inside the 2DEG is effectively one-dimensional. Assuming that reflection at the interfaces between the semiconducting bridge and the superconducting reservoirs is specular (i.e., electrons from different modes do not mix and, therefore, can be treated independently), we can describe each mode with a simplified one-dimensional model [15]. We assume that the left and right superconductors have uniform order parameters of the same magnitude $\Delta$, but with different phases, $\phi_{1}$ and $\phi_{2}$. The order parameter inside the semiconducting bridge vanishes, i.e.

$$
\Delta(x)=\left\{\begin{array}{lr}
\Delta \mathrm{e}^{i \phi_{1}} & x<-L / 2, \\
0, & -L / 2<x<L / 2, \\
\Delta \mathrm{e}^{i \phi_{2}} & x>+L / 2 .
\end{array}\right.
$$

In our model we neglect interelectron interactions inside the 2DEG. For such a quasi-1D constriction we could have used a Luttinger liquid model to describe the one-dimensional transverse channels between the superconductors (see, e.g., Refs. 16 and 17). It turns out, however, that for an adiabatic constriction both models produce the same final result.

The influence of a magnetic field on the electrons in the 2DEG is twofold. First, the magnetic field acts on the magnetic moments of the electrons and holes, and second it affects the spatial motion of the charge carriers via the Lorentz force. However, if the magnetic field is applied parallel to the plane of the 2D electron gas, orbital effects can be neglected. In this article we consider only the effects of Zeeman interactions.

We assume that the properties of the 2DEG/S interface are very similar to the properties of a normal (N) metal/superconductor $(\mathrm{S}), \mathrm{N}-\mathrm{S}$, interface, namely, the electrons and holes from the nonsuperconducting part of the constriction, whose energies are lower than the energy gap $\Delta$ in the spectrum of the superconductors, cannot leave the normal part of our S/2DEG/S junction. Instead they are being Andreev reflected [18] at the 2DEG/S interfaces. The interference of the incident electron and the reflected hole produces a set of discrete Andreev-Kulik (AK) states [19], that are responsible for the occurence of a Josephson current through the system. The spectrum of the AK states can be found by solving the BogolyubovDe Gennes equations [15] 


$$
\left(\begin{array}{cc}
\hat{\mathbf{H}}(x) & \hat{\mathbf{D}}(x) \\
\hat{\mathbf{D}}^{*}(x) & -\hat{\mathbf{H}}(x)
\end{array}\right)\left(\begin{array}{c}
\mathbf{u}(x) \\
\mathbf{v}(x)
\end{array}\right)=\varepsilon\left(\begin{array}{c}
\mathbf{u}(x) \\
\mathbf{v}(x)
\end{array}\right)
$$

where the two-component spinors $\mathbf{u}(x)=\left(u_{\uparrow}(x), u_{\downarrow}(x)\right)$ and $\mathbf{v}(x)=\left(v_{\uparrow}(x), v_{\downarrow}(x)\right)$ are, respectively, electron and hole probability amplitudes, and the $\uparrow$ and $\downarrow$ subscripts determine the quasiparticle spin projection. The Hamiltonian $\hat{\mathbf{H}}$ is a $2 \times 2$ matrix

$$
\hat{\mathbf{H}}(x)=h_{e}\left(\begin{array}{ll}
1 & 0 \\
0 & 1
\end{array}\right)+\mu\left(\begin{array}{cc}
1 & 0 \\
0 & -1
\end{array}\right) .
$$

Here $h_{e}=p^{2} / 2 m(x)+U(x)-\varepsilon_{F}$ and $\mu=g \mu_{B} / 2$. The first term in Eq. (3) describes the nonmagnetic part of the Hamiltonian with $\varepsilon_{F}$ being the Fermi energy, and $m(x)$ the effective mass of the quasiparticles. The effective mass $m_{s}$ in the superconductors can be different from the effective mass $m_{n}$ in the semiconductor. The function $U(x)$ describes the external potential. We assume that the $\mathrm{S} / 2 \mathrm{DEG} / \mathrm{S}$ junction is clean and, therefore, $U(x)=0$ everywhere except at the interfaces. At the interfaces $(x= \pm L / 2)$ the difference in material composition of superconductor and semiconductor commonly leads to formation of potential barriers. We will model these potential barriers by Dirac delta-functions of equal strength $W$, i.e.

$$
U(x)=W(\delta(x+L / 2)+\delta(x-L / 2)) .
$$

The second term in Eq. (3) is the Zeeman coupling of the magnetic momenta of the electrons to the external field $B(x)$. We assume that the magnetic field is weak $\left(g \mu_{B} B(x)<<\Delta\right.$, where $\mu_{B}$ is the Bohr magneton and $g$ is the Lande $g$-factor), and that it acts only in the nonsuperconducting part of the junction

$$
B(x)=\left\{\begin{array}{l}
B, \text { if }|x|<L / 2, \\
0, \text { otherwise }
\end{array}\right.
$$

These requirements are important in order to guarantee that the field does not create currents (or equivalently, phase gradients) along the S/2DEG interfaces. Finally, the off-diagonal potential $\hat{\mathbf{D}}(x)$, which enters Eq. (2) is equal to

$$
\hat{\mathbf{D}}(x)=\Delta(x)\left(\begin{array}{ll}
1 & 0 \\
0 & 1
\end{array}\right),
$$

where $\Delta(x)$ is the order parameter, Eq. (1). The use of the Bogolyubov-de Gennes equations to describe a S/2DEG/S structure is valid only when the nonsuperconducting segment of the system is shorter than the electron-hole pair correlation decay length $l_{T}=\hbar v_{F} /\left(k_{B} T\right)$ (see, e.g., discussion in Ref. 20). The Andreev-Kulik levels are obtained by matching the solutions for the uniform regions at the S/2DEG interfaces. The result of the matching procedure is quite cumbersome. To simplify it we will use the Andreev approximation $[18,19]$. That is, to the lowest nonvanishing order in $\max (\Delta, E) / \varepsilon_{F}$ we (approximately) replace the wave vectors of the electrons and holes inside the $\mathrm{S}$ and 2DEG parts of the junction with the Fermi wave vectors $k_{e} \approx k_{h} \approx k_{F}^{(n)}$ and $q_{e} \approx q_{h} \approx k_{F}^{(s)}$, where $k_{F}^{(s)} / k_{F}^{(n)}=\sqrt{m_{s} / m_{n}}$; and for the differences between the wave vectors we write $k_{e}-k_{h} \approx m_{n} \varepsilon /\left(\hbar^{2} k_{F}^{(n)}\right)$ and $q_{e}-q_{h} \approx m_{s} \Delta /\left(\hbar^{2} k_{F}^{(s)}\right)$.

In a long junction $\left(L>>\xi_{0}=\hbar v_{F} / \Delta\right)$ the bound state energies close to the Fermi level, $\varepsilon<<\Delta$, are

$$
\varepsilon_{n, \sigma}^{( \pm)}(\phi)=\pi \omega_{L}\left( \pm \frac{\Theta}{2 \pi}+n\right)+\frac{\sigma}{2} g \mu_{B} B,
$$

where the new phase $\Theta$ is defined as

$$
\begin{aligned}
& \Theta \approx \pi-\arccos \left(\frac{R_{1} \cos (\phi)+R_{2} \cos \left(2 L k_{F}\right)+R_{3} \sin \left(2 L k_{F}\right)}{D_{Z}}\right), \\
& D_{Z}=\left[m_{s}\left(m_{n}+m_{s}\right)+4 m_{n}^{2} Z^{2}\right]^{2}, R_{1}=4 m_{n} m_{s}^{3}, \\
& \left.R_{2}=-\left[\left(m_{n}-m_{s}\right)^{2} m_{s}^{2}+8 m_{n}^{2}\left(m_{n}-3 m_{s}\right) m_{s} Z^{2}+16 m_{n}^{4} Z^{4}\right)\right], \\
& R_{3}=8 m_{n} m_{s} Z\left[\left(m_{n}-m_{s}\right) m_{s}+4 m_{n}^{2} Z^{2}\right] .
\end{aligned}
$$

Here $Z=W m /\left(\hbar k_{F}\right)$ is the parameter which describes the barrier strength and $\omega_{L}=\hbar v_{F} / L$ is the energy level spacing. If the effective masses of the charge carriers in the superconducting leads and in the $2 \mathrm{DEG}$ are equal, the results obtained in Refs. 21-23 are recovered. Equation (6) describes two sets ( \pm ) of discrete levels, labeled by integer indices $n=0, \pm 1, \pm 2, \ldots$ and an additional index $\sigma= \pm 1$ which characterizes the splitting of the energy levels in a magnetic field. Since the scattering properties of the interfaces are assumed to be spin-independent, spin is conserved in both the Andreev and normal reflections. The magnetization of each populated AK level is, according Eq. (6), $g \mu_{B} / 2$. The mesoscopic harmonic factors, $\sin \left(2 L k_{F}\right)$ and $\cos \left(2 L k_{F}\right)$ in Eq. (7), are associated with interference of the incident and normally reflected quasiparticle waves. They appear due to a large change in the quasimomentum of the quasiparticle at the interface in normal reflection. In a transparent SNS junction $(Z=0)$ with equal effective masses, these oscillations are absent since in pure Andreev reflection processes momentum is 
approximately conserved. As a result, Eq. (6) reduces to the well known Kulik spectrum [19].

For a transparent SNS junction in zero magnetic field, $B$, the AK levels, corresponding to two different sets $( \pm \phi)$, intersect at $\phi_{r}=\pi+2 \pi r(r=0, \pm 1, \pm 2, \ldots)$. At these special points the levels are four-fold degenerate for a single channel junction. The situation is changed if the junction is not transparent. Even if the barriers are very small, levels do not intersect and they oscillate periodically with phase, approaching each other at the points $\phi=\pi k(k=0, \pm 1, \pm 2)$. Every AK level is now two-fold degenerate. An external magnetic field removes the remaining degeneracy. The magnetization generated by the AK state $n \sigma( \pm)$ of the transverse mode $l$ is given by

$$
M_{l, \sigma}^{( \pm)}(n)=-\frac{\mu \sigma}{1+\exp \left(-\beta \varepsilon_{l, n, \sigma}^{( \pm)}(\phi)\right)} .
$$

The subscript $l$ which enters this formula implies that the transverse modes are different. The total magnetization of a single transverse mode is given by a sum over all the AK states.

It is useful to define the magnetizations produced by pairs of levels with opposite directions of the magnetic moment $M_{l}^{( \pm)}(n)=M_{l,+1}^{( \pm)}(n)+M_{l,-1}^{( \pm)}(n)$ and then sum up the pair contributions $M_{l}^{( \pm)}(n)$ over the index $n$ using the Poisson summation formula

$$
\begin{aligned}
& \sum_{n=-\infty}^{+\infty} M_{l}^{( \pm)}(n)= \\
& =\int_{-\infty}^{\infty} M_{l}^{( \pm)}(v) d v+2 \operatorname{Re}\left\{\sum_{k=1}^{+\infty} \int_{-\infty}^{\infty} M_{l}^{( \pm)}(v) \mathrm{e}^{2 \pi i k v} d v\right\} .
\end{aligned}
$$

Such ordering guarantees convergence of the first and second terms in Eq. (9). The total magnetization of the single transverse mode is [8]

$$
M_{l}=\frac{g^{2} \mu_{B}^{2} B}{\pi \omega_{L}}+\frac{4 T g \mu_{B}}{\omega_{L}} \sum_{k=1}^{\infty} \frac{\cos (k \Theta) \sin (k \chi)}{\sinh \left(2 \pi k T / \omega_{L}\right)},
$$

where $\chi=g \mu_{B} B / \omega_{L}$.

The first term in Eq. (10) does not depend on the phase difference and the temperature. This term describes magnetization of the junction in the absence of Andreev reflection; that is, for nonsuperconducting reservoirs (Pauli magnetization). It is the second term which is responsible for superconductivity-induced oscillations of the magnetization. At high temperatures $\left(T \gg>\omega_{L}\right)$, when the Fermi distribution is smeared over many AK levels the amplitude of these oscillations is small in comparison with the first term in Eq. (10). The sum in Eq. (10) can be truncated at $k=1$ so that the magnetization of each trans- verse mode oscillates as $A \cos \Theta$ with an amplitude $A=\left(4 g^{2} \mu^{2} B T / \omega_{L}^{2}\right) \exp \left(-2 \pi T / \omega_{L}\right)$.

In the opposite limiting case, at low temperatures $\left(T<g \mu_{B} B\right)$, only a small number of states near the Fermi energy contributes to the magnetization. The AK levels are shifted in energy because of the change of the phase difference $\phi$ between the superconductors. At certain values of the phase difference some AK states approach very close to the Fermi energy. At these phase differences the magnetization of the junction will increase.

Taking into account only a single pair of states which is the closest (at the given $\phi=\phi_{r}$ ) to the Fermi energy and neglecting the contribution of the other states, we can approximate the previous formula by a simple expression

$$
\begin{aligned}
& M_{l}^{(\mathrm{osc})} \approx \frac{g \mu_{B}}{\exp \left(\frac{\omega_{L}}{2 T} \Theta-\frac{g \mu_{B}}{2 T} B\right)+1}- \\
& -\frac{g \mu_{B}}{\exp \left(\frac{\omega_{L}}{2 T} \Theta+\frac{g \mu_{B}}{2 T} B\right)+1},
\end{aligned}
$$

where the angle $\Theta$ is defined by Eq. (7). The same result can be achieved if we use the Euler-Maclaurin summation formula to approximate Eq. (10) in the vicinity of the points $\phi_{r}=\pi(1+2 r)$.

Formally Eq. (10) describes only the part of the magnetization that is due to the discrete spectrum of $\mathrm{AK}$ states. The continuous spectrum $(|\varepsilon|>\Delta)$ also contributes to the magnetization [24]. At temperatures $T \ll \Delta$ the effect of continuous spectrum is to compensate the nonsmooth contributions of the discrete spectrum arising when summing up to the finite number of bound states inside the energy interval $|\varepsilon|<\Delta$. In our calculation method (see Eqs. (9) and (10)) the superconducting gap was formally put equal to infinity, and as a result only the discrete spectrum survives. This method reproduces the correct results for thermodynamic properties of a long SNS junction at temperatures $T \ll \Delta$ (see the corresponding discussion in a recent review [25]).

In order to evaluate the total magnetization of the junction we have to sum up the contributions of all the open transverse modes (channels). A channel is open if the energy of its lowest longitudinal mode is smaller than the Fermi energy of the superconducting leads that it is connected to. Since the barriers at the S/2DEG interfaces are assumed small the motion in the longitudinal direction is almost unperturbed. This situation corresponds to the case of junctions fabricated in InGaAs heterostructures with $\mathrm{Nb}$ electrodes [9]. Confinement in the lateral direction is usually created by an electrostatic potential generated by gate electrodes etched on the surface of the semiconductor. 
We obtained results for two simple models of the confining potential: i) a hard wall potential, and ii) a parabolic potential. For more accurate description one needs to solve the Schrödinger and Poisson equations self-consistently as described in Refs. 26 and 27.

For hard wall boundary conditions, we can express the velocity of the electrons and the holes, with energies near the Fermi energy in the $l$ th channel, in the form

$$
v_{F}^{(l)}=\sqrt{\frac{2}{m_{n}}\left(\varepsilon_{F}-\frac{1}{2 m_{n}}\left(\frac{\hbar \pi l}{d}\right)^{2}\right)} .
$$

The velocities of quasiparticles in a multichannel junction with parabolic confinement $U(y)=m_{n} \Omega_{\perp}^{2} y^{2} / 2$ are

$$
v_{F}^{(l)}=\sqrt{\frac{2}{m_{n}}\left(\varepsilon_{F}-\hbar \Omega \perp\left(l+\frac{1}{2}\right)\right)},
$$

where the lateral frequency $\Omega_{\perp}=\sqrt{8 \varepsilon_{F} /\left(m_{n} d^{2}\right)}$ was chosen such that $U(y= \pm d / 2)=\varepsilon_{F}$.

From this formula we see that the velocities and, consequently, the separation between the AK levels $\omega=\omega_{l}=\hbar v_{F}^{(l)} / L$ are different for different transverse modes. If the $2 \mathrm{DEG}$ junction is wide the number of modes is large and we can replace the summation over them by an integration and write the magnetization of the $\mathrm{S} / 2 \mathrm{DEG} / \mathrm{S}$ junction as

$$
M \approx N_{\perp} g \mu_{B} \int_{0}^{1} D(\xi, \alpha, \beta) \lambda(\xi) d \xi .
$$

The function $D(\xi, \alpha, \beta)=f(\alpha \xi-\beta)-f(\alpha \xi+\beta)$ is the difference between the occupation of the two states with opposite momenta, $f(\eta)=1 /\left(\mathrm{e}^{\eta}+1\right)$ is the Fermi distribution, $\alpha=\hbar v_{F} \Theta /(2 L T)$ and $\beta=g \mu_{B} B /(2 T)$. The weight function $\lambda(\xi)$ is equal to $2 \xi$ for the «soft walls» potential and to $\xi / \sqrt{1-\xi^{2}}$ for the «hard walls» potential.

The integral on the right hand side of Eq. (14) cannot be calculated analytically, but we can find the asymptotic behavior of the magnetization in the vicinity of several values of the of phase difference $\phi$. In the vicinity of the resonance points $\phi_{r}$ where $\Theta<L L T /\left(\hbar v_{F}\right)$, the magnetization is the highest, and it is approximately equal to

$$
\begin{aligned}
& M \approx N_{\perp} g \mu_{B} \tanh \left(g \mu_{B} B / 4 T\right) \times \\
& \times\left[1-\frac{C_{1}}{12}\left(\frac{\Theta \hbar v_{F}}{L T}\right)^{2} \operatorname{sech}^{2}\left(g \mu_{B} B / 4 T\right)\right] .
\end{aligned}
$$

The constant $C_{1}$ is equal to $1 / 2$ in the «hard wall» potential and to $2 / 3$ in the «soft wall» potential model. On the other hand, if the phase is far away from resonance $\left(\Theta>>L T /\left(\hbar v_{F}\right)\right)$ the magnetization approaches slowly the value

$$
M \approx C_{2} N_{\perp} g^{2} \mu_{B}^{2} B T\left(\frac{2 L}{\hbar v_{F} \Theta}\right)^{2} \ln 2 .
$$

The constant $C_{2}$ is equal to 1 for the «hard wall» model and it takes the value 2 for the «soft wall» model. While Eq. (15) works well for transparent $S / 2 D E G / 2$ junction, it may be inaccurate if the barriers at the interfaces are not small enough. In this case the condition $\Theta<<1$ cannot be satisfied at any phase $\phi$.

In Fig. 2 we show the result of numerical calculations for the magnetization as a function of the phase difference, and compare this numerical result with the asymptotic behavior in Eqs. (15) and (16). Figure 3 shows the behavior of the magnetization at several different temperatures. Higher temperatures lead to smearing of the Fermi distribution and the resonance peaks become smaller and broader.

Since for transparent clean junction the resonance condition $\phi=\phi_{r}$ is the same for all transverse modes, magnetization at the resonances is proportional to the number of transverse modes. This effect is analogous to the giant oscillations of the conductance considered in Ref. 28. Therefore, the wider the junction, the higher would be the magnetization at the resonances. This behavior is illus-

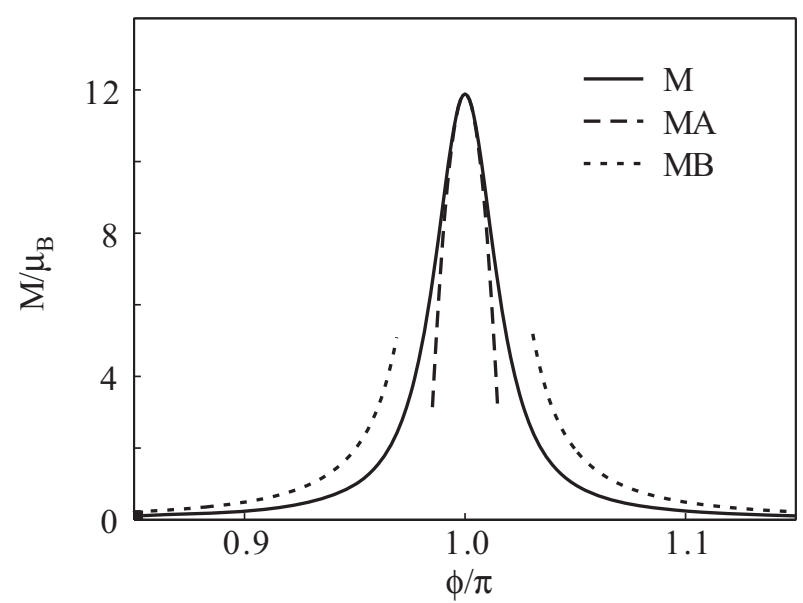

Fig. 2. Magnetization of a transparent $(Z=0) \mathrm{S} / 2 \mathrm{DEG} / \mathrm{S}$ junction with a harmonic (parabolic) lateral confining potential in a magnetic field $B=10 \mathrm{Oe}$, plotted as a function of the phase difference. Also shown are its asymptotes MA (Eq. (15)) and MB (Eq. (16)). For this demonstration we choose materials with the same effective masses. We assume that the superconductors are made from niobium, with a Fermi energy $\varepsilon_{F}(\mathrm{Nb})=8.52 \cdot 10^{-12} \mathrm{erg}$, and effective masses $m_{S}=m_{n}$. The length of the 2DEG part of the junction is $L=10^{-4} \mathrm{~cm}$, and it's width is $d=6 \cdot 10^{-5} \mathrm{~cm}$. Results are shown for a temperature $T=0.1 \mathrm{~K}$, which is much lower than the critical temperature of niobium $T_{C}(\mathrm{Nb})=9.2 \mathrm{~K}$. Note that the resonance peaks are extremely narrow, their width $\delta \phi \sim g \mu_{B} B / \omega_{L}$ is approximately equal to the phase change necessary to shift the spectrum of AK levels by an amount equal to Zeeman splitting. 


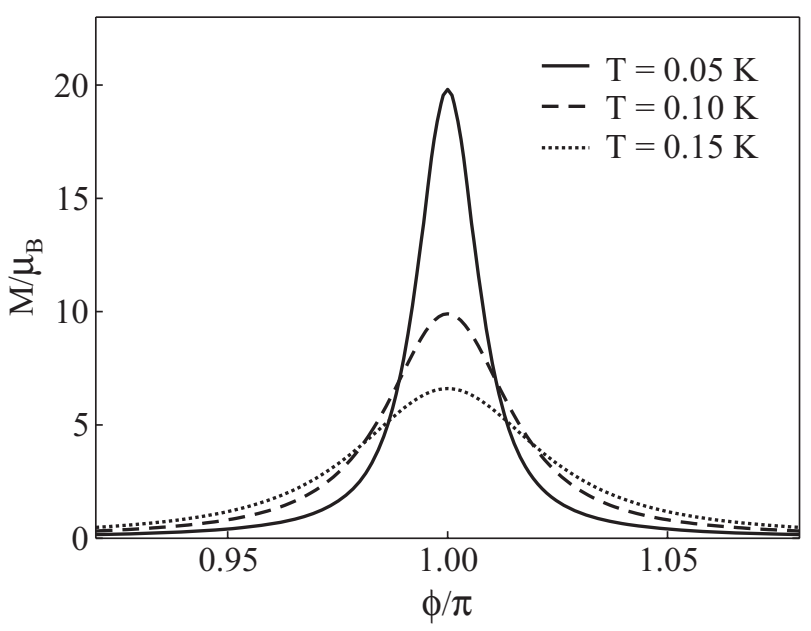

Fig. 3. Magnetization of the transparent $\mathrm{S} / 2 \mathrm{DEG} / \mathrm{S}$ junction in a magnetic field $B=10 \mathrm{Oe}$ at several temperatures plotted versus the phase difference. The width of the junction is $d=5 \cdot 10^{-5} \mathrm{~cm}$, and all the other parameters of the junction are as in Fig. 2.

trated in Fig. 4. On the other hand, varying the length of the junction will affect only the width of the peaks, whereas their height will remain unchanged (see Fig. 5).

In the case of nontransparent junctions, the electrons and holes incident on the surface may be reflected normally from the $\mathrm{S} / 2 \mathrm{DEG}$ interface. This process modifies the spectrum of the AK levels. The AK levels do not intersect and do not cross the Fermi energy at any value of the phase $\phi$. As a consequence, the amplitude of the magnetization peaks decreases. Differences between the effective masses of the 2DEG and the superconductors have a similar effect on the magnetization as the presence of barriers at the interfaces. Namely, because of the mismatch be-

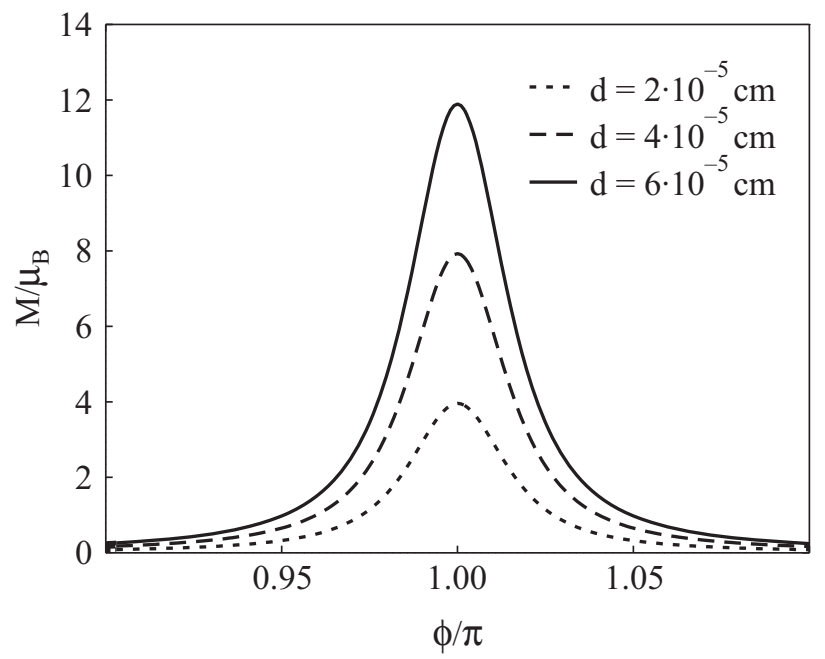

Fig. 4. A magnetization as a function of the width of the $2 \mathrm{DEG}$ part of a transparent $\mathrm{S} / 2 \mathrm{DEG}$ junction. The length of the junction is $L=10^{-4} \mathrm{~cm}$ and the temperature $T=0.1 \mathrm{~K}$. The strength of the magnetic field and all other parameters are the same as in Fig. 2.

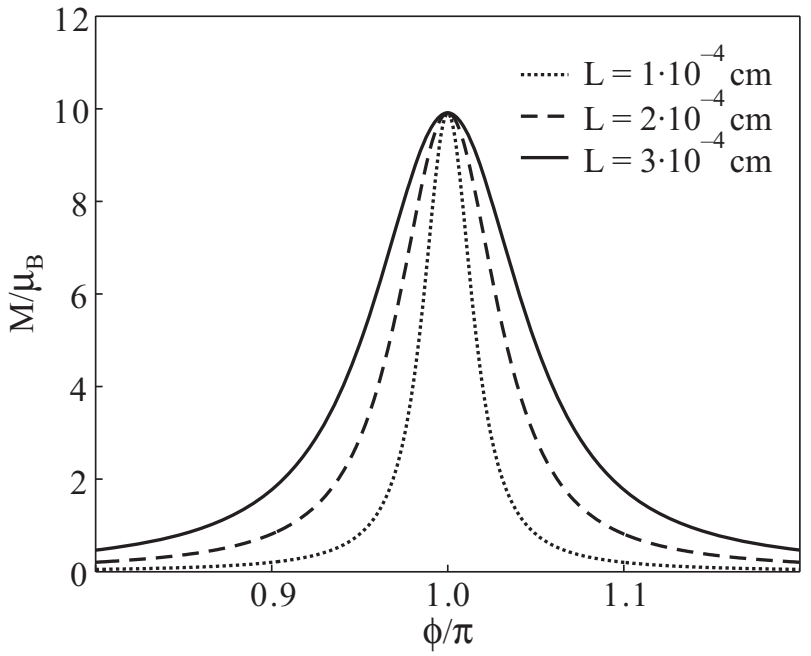

Fig. 5. The magnetization of a transparent S/2DEG/S junction versus the phase difference $\phi$, for different lengths of the 2DEG part. The width of the junction is $d=5 \cdot 10^{-5} \mathrm{~cm}$, temperature $T=0.1 \mathrm{~K}$ and the magnetic field $B=10 \mathrm{Oe}$.

tween the Fermi velocities some of the electrons are being normally reflected at the 2DEG/S surface. For larger difference between the effective masses, larger fraction of all of the incident electrons will be reflected from the interfaces normally.

In clean $\mathrm{S} / 2 \mathrm{DEG} / \mathrm{S}$ or $\mathrm{S} / \mathrm{N} / \mathrm{S}$ junctions every Andreev reflected electron picks up an additional phase $\delta \phi= \pm \phi_{1,2}+\arccos (E / \Delta)$ at the interface, whereas normal electrons do not pick up such a phase. The wave function of the reflected electron-hole pair is a mixture of Andreev and normally reflected electrons and holes. The resonance conditions for this mixture are different from the resonance conditions for the wave function in the clean S/2DEG/S junction, where only Andreev reflection can occur. The resonance conditions in the junction with barriers, or with different effective masses, depend not only on the phase difference, but also on the length of the junction. Although it is always possible to achieve resonance for one channel, it is difficult to satisfy the resonance conditions for many channels simultaneously, since different transverse channels have different longitudinal velocities. The resonant peaks for magnetization of each single channel are very narrow, with the majority of the channels being off resonance even for small barriers or slightly different materials. As a result, the total magnetization of the junction for both barrier models (hard and soft walls) and for different masses, is strongly suppressed. Therefore, for observation of strong resonance behavior the use of materials with similar effective masses is recommended.

The magnetization, as well as the superconducting current in a S/2DEG/S junction, result from the difference in the population of the different AK levels. Josephson current through the junction is possible only when the en- 
ergy levels of the two sets $\left(d E_{n} / d \phi>0\right.$ and $\left.d E_{n} / d \phi<0\right)$ are unequally populated. Similarly, magnetization of the $\mathrm{S} / 2 \mathrm{DEG} / \mathrm{S}$ junction is a result of different population of states with opposite directions of the magnetic moments. It is most interesting to note that the magnetization is more sensitive to the barriers and mass differences than the Josephson current. Indeed, since we are constrained to use weak external magnetic fields in order to avoid destruction of superconductivity, Zeeman splitting of the AK levels is small $\left(\mu_{B} B=\Delta_{Z}<<\omega_{L}\right)$. In this situation, impurities, barriers, or effective mass difference that modify the spectrum of the AK levels can move them away from the region $(\sim T)$ where the gradient of the Fermi distribution is high. Even a small shift $\Delta_{b}$ of the energy levels from the Fermi level may result in the situation $T \ll \Delta_{Z} \ll \Delta_{b}$ when both levels with opposite direction of the magnetic moment are almost equally populated, combining to give a small magnetization $M_{l} \ll \mu_{B}$. This situation does not occur for levels which belong to different sets. These levels are well separated $\sim \Delta_{L}$ and small change $\Delta_{b} \ll \Delta_{L}$ in their positions cannot affect their population significantly.

\section{Differences between properties of $S / 2 D E G / S$ and S/GM/S junctions}

We turn now to analysis of a junction with the geometry similar to that of the $\mathrm{S} / 2 \mathrm{DEG} / \mathrm{S}$ junction dicussed above, but where the two superconducting leads are connected to each other through a monolayer of graphene. Graphene attracted much recent attention. In this material the dispersion relation for the low-energy electrons and holes is similar to the dispersion relation of relativistic massless fermions. This quasi-relativistic behavior of the electrons in graphene has many interesting consequences. One of these is the existence of two types of Andreev reflections at the graphene/superconductor boundary [29]. Another consequence is the effect of Klein tunneling through the a potential barrier [30]. One might expect that Klein tunneling and specular Andreev reflection would enhance magnetic effects by reducing the strong destructive interference between normally and Andreev reflected electrons and holes in the nonsuperconducting (graphene) part of the junction. In addition to specular Andreev reflection and Klein tunneling one should also take into account the fact that reflection of the electrons from the edges of the graphene ribbon depends on the orientation of the crystallographic axes with respect to the line of the edge at the sides of the graphene ribbon - this affects the quantization of the transverse motion within the ribbon [31]; in our calculations we assumed infinite-mass boundary conditions at the edges, and as a result our transverse momentum is quantized as $q_{l}=(l+1 / 2) \pi / W$.

Similar to the S/2DEG/S junction, by matching the solutions of the Schrödinger equation for each part of the contact (see Ref. 32) one can find the spectrum of the AK levels in the $\mathrm{S} / \mathrm{GM} / \mathrm{S}$ contact and use this spectrum to calculate the total magnetization of the $\mathrm{S} / \mathrm{GM} / \mathrm{S}$ junction under the same conditions as for the $\mathrm{S} / 2 \mathrm{DEG} / \mathrm{S}$ one. It turns out, however, that the magnetization of the S/GM/S junction is very small even in the absence of potential barriers at the interfaces between the superconductor leads and
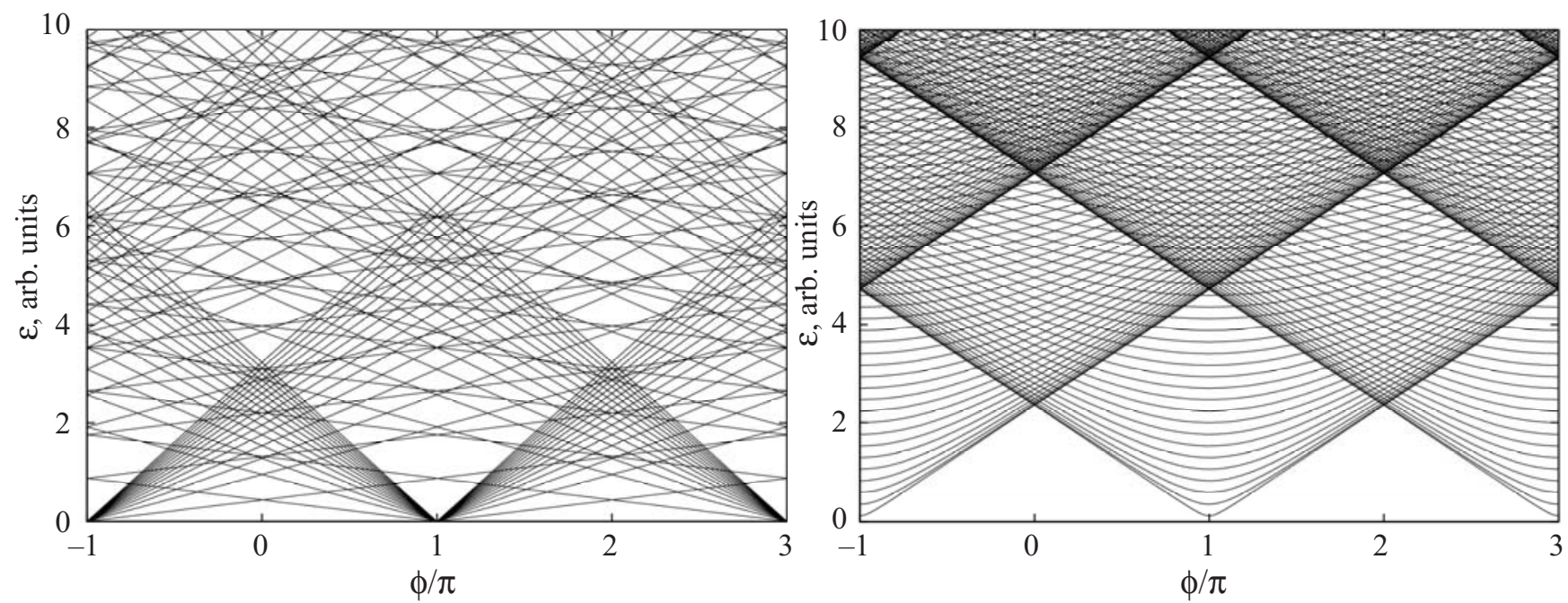

Fig. 6. The spectrum of AK levels in a S/2DEG/S junction (left) and in a S/GM/S junction (right). Both figures are schematic, that is, these figures are plotted for junctions with different geometrical parameters with the main purpose to illustrate the basic characteristic features of the energy levels in these systems. Note, that unlike the S/2DEG/S junction where the lowest AK levels of all transverse modes approach the Fermi level and become degenerate at the resonant phases $\left(\phi_{r}=\pi+2 \pi r, r=0, \pm 1, \pm 2, \ldots\right)$, the lowest AK levels of the $\mathrm{S} / \mathrm{GM} / \mathrm{S}$ junction, belonging to different modes, never become degenerate at the resonant phases, and all (except one level of the lowest transverse mode) stay away from the Fermi level. 
the graphene. In fact the magnetization is much smaller than that found for the $\mathrm{S} / 2 \mathrm{DEG} / \mathrm{S}$ junction (see above). There are several reasons for such a small effect. First is the fact that the density of states of graphene near the Fermi level (for undoped graphene) is very small and therefore the number of AK levels in the range of energies near the Fermi level for the S/GM/S junction should be much smaller than for the S/2DEG/S junction. The other reason for the smallness of magnetization is that the $\mathrm{AK}$ levels in different transverse modes of the $\mathrm{S} / \mathrm{GM} / \mathrm{S}$ junction behave differently (see Fig. 6) and they do not converge to a single degenerate state at the Fermi level for the resonant phase differences, as happens for $\mathrm{S} / 2 \mathrm{DEG} / \mathrm{S}$ junctions (see above). Since the energy levels of the transverse modes in the $\mathrm{S} / \mathrm{GM} / \mathrm{S}$ junction never approach the Fermi level and always stay away from the region with maximal gradients of the Fermi distribution, their contribution to the total magnetization is very small.

We conclude that the different behavior of the AK levels in the semiconductor 2DEG and in graphene originate from the different type of dispersion relations of the electrons in the two cases: a quasi-relativistic linear dispersion relation for the electrons in graphene versus the regular quadratic dispersion relation in the two-dimensional electron gas.

\section{Summary}

In summary, we considered magnetic effects in a two-dimensional electron gas bridging two superconducting reservoirs. We demonstrated that this system can exhibit interesting superconductivity-induced magnetic resonance effects. Namely, we predict sharp increases in the magnetic susceptibility of the junction at special values $\phi_{r}=\pi+2 \pi r$ of the phase difference $\phi=\phi_{1}-\phi_{2}$ of the order parameter between the two superconductors.

This effect results from a change of the population of the AK levels near the Fermi energy. In general, magnetization of a single transverse mode due to the Andreev levels is very small but since for transparent junctions the resonance conditions are identical for all transverse modes, the magnetic response at the resonances (at low temperatures) is proportional to the number of transverse modes. Consequently, when the number of transverse modes is large, the total magnetization of the junction may become large enough to allow experimental detection.

We also considered a junction made from a graphene ribbon bridging the two superconductors. We found that the giant magnetization oscillations that we predict for the $\mathrm{S} / 2 \mathrm{DEG} / \mathrm{S}$ junction are absent in the Josephson contact made with the monolayer graphene ribbon. Reasons for the differences in magnetization response between the $\mathrm{S} / 2 \mathrm{DEG} / \mathrm{S}$ and $\mathrm{S} / \mathrm{GM} / \mathrm{S}$ systems were discussed.
The research of I.A.R., E.N.B., and U.L. was supported by the U.S. Department of Energy, grant No. FG-05-86ER 45234.

I.V.K. acknowledges the financial support from the grant «Effects of electronic, magnetic and elastic properties in strongly inhomogeneous nanostructures» by the National Academy of Sciences of Ukraine and the financial support from the Royal Swedish Academy of Sciences.

1. B.J. van Wees, H. van Houten, C.W.J. Beenakker, J.G. Williamson, L.P. Kouwenhoven, D. van der Marel, and C.T. Foxon, Phys. Rev. Lett. 60, 848 (1988).

2. D.A. Wharam, T.J. Thornton, R. Newbury, M. Pepper, H. Ahmed, J.E.F. Frost, D.G. Hasko, D.C. Peacock, D.A. Ritchie, and G.A.C. Jones, J. Phys. C21, L209 (1988).

3. U. Landman, W.D. Luedtke, N. Burnham, and R.L. Colton, Science 248, 454 (1990).

4. N. Agrait, A.L. Yeyati, and J.M. van Ruitenbeek, Phys. Rep. 377, 8103 (2003).

5. E.N. Bogachek, A.M. Zagoskin, and I.O. Kulik, Fiz. Nizk. Temp. 16, 1404 (1990) [Sov. J. Low Temp. Phys. 16, 796 (1990)].

6. A.G. Scherbakov, E.N. Bogachek, and U. Landman, Phys. Rev. B53, 4054 (1996).

7. Xiaolin Li, Xinran Wang, Li Zhang, Sangwon Lee, and Hongjie Dai, Science 29, 1229 (2008).

8. I.V. Krive, I.A. Romanovsky, E.N. Bogachek, and U. Landman, Phys. Rev. Lett. 92, 126802 (2004).

9. H. Takayanagi, T. Akazaki, and J. Nitta, Phys. Rev. Lett. 75, 3533 (1995).

10. S.G. den Hartog, B.J. van Wees, Yu.V. Nazarov, T.M. Klapwijk, and G. Borghs, Phys. Rev. Lett. 79, 3250 (1997).

11. H.B. Heersche, P. Jarillo-Herrero, J.B. Oostinga, L.M.K. Vandersypen, and A.F. Morpurgo, Nature 446, 56 (2007).

12. F. Miao, S. Wijeratne, Y. Zhang, U.C. Coskun, W. Bao, and C.N. Lau, Science 317, 1530 (2007).

13. Supriyo Datta, Electronic Transport in Mesoscopic Physics, Cambridge University Press, New York (1995).

14. E.V. Bezuglyi, A.S. Rozhavsky, I.D. Vagner, and P. Wyder, Phys. Rev. B66, 052508 (2002).

15. P.G. de Gennes, Superconductivity of Metals and Alloys, Addison-Wesley, New York (1992).

16. D.L. Maslov, M. Stone, P.M. Goldbart, and D. Loss, Phys. Rev. B53, 1548 (1996).

17. J.S. Caux, H. Saleur, and F. Siano, Phys. Rev. Lett. 88, 106402 (2002).

18. A.F. Andreev, Sov. Phys. JETP 19, 1228 (1964).

19. I.O. Kulik, Sov. Phys. JETP 30, 944 (1970).

20. A.M. Zagoskin, Quantum Theory of Many-Body Systems, Springer, New York (1998).

21. G.A. Gogadze and A.M. Kosevich, Fiz. Nizk. Temp. 24, 716 (1998) [Low Temp. Phys. 24, 540 (1998)].

22. O. Cakir and I.O. Kulik, Phys. Rev. B67, 174514 (2003).

23. P.F. Bagwell, Phys. Rev. B46, 12573 (1992).

24. C.W. Beenakker and H. van Houten, Phys. Rev. Lett. 66, 3056 (1991). 
25. I.V. Krive, S.I. Kulinich, R.I. Shekhter, and M. Jonson, Fiz. Nizk. Temp. 30, 738 (2004) [Low Temp. Phys. 30, 554 (2004)].

26. S.E. Laux, D.J. Frank, and F. Stern, Surface Science 196, 101 (1988).

27. J.H. Davies and J.A. Nixon, Phys. Rev. B39, 3423 (1989).

28. A. Kadigrobov, A.M. Zagoskin, R.I. Shekhter, and M. Jonson, Phys. Rev. B52, R8662 (1995).
29. C.W. Beenakker, Phys. Rev. Lett. 97, 067007 (2006).

30. M.I. Katsnelson, K.S. Novoselov, and A.K. Geim, Nature Physics 2, 620 (2006).

31. J. Tworzydlo, B. Trauzettel, M. Titov, A. Rycerz, and C.W.J. Beenakker, Phys. Rev. Lett. 96, 246802 (2006).

32. M. Titov and C.W. Beenakker, Phys. Rev. B74, 041401(R) (2006). 